\title{
High Cholesterol, Triglycerides, and Body-Mass Index in Suicide Attempters
}

Jürgen Brunner, Thomas Bronisch, Hildegard Pfister, Frank Jacobi, Michael Höfler, and Hans-Ulrich Wittchen

Low cholesterol concentrations and cholesterol-lowering therapies have been suggested to be associated with increased suicidality. This article examined the association of cholesterol, triglycerides, and body-mass index (BMI) with suicidal ideation and suicide attempts. Findings are based on a nationally representative community sample of $n=4,181$ subjects ( $18-65$ years) examined with a standardized diagnostic interview (CIDI) for (DSM-IV) mental disorders. Controlling for age and gender the study revealed a moderate positive association between cholesterol, triglycerides, BMI, and suicide attempts in subjects with depressive symptoms during the past 12 months $(n=1,205)$. The results of this study are compatible with two recent epidemiological cohort studies showing a positive association between cholesterol and completed suicide.

Keywords major depressive episode, suicide attempt, cholesterol, body-mass index

A meta-analysis of selected cholesterol-lowering trials revealed an unanticipated association between trial-reduced serum cholesterol concentrations and increased mortality from suicide, accidents, trauma, and violence (Muldoon, Manuck, Matthews et al., 1990). Based on these findings it has been speculated that cholesterol-lowering therapies may cause or worsen depressive symptoms and increase the risk of suicide. However, this meta-analysis has to be regarded as inconclusive due to several methodological limitations (review: Brunner, Parhofer, Schwandt et al., 2002). A recently published meta-analysis of cholesterol-lowering therapies (Muldoon, Manuck, Mendelsohn et al., 2001) including more than 70,000 subjects showed no increase of mortality from suicide, accidents, and violence. Statins significantly reduce cardiovascular morbidity and mortality (LaRosa, He, \& Vupputuri, 1999) without increasing mortality from suicide or trauma (Muldoon, Manuck, Mendelsohn et al., 2001).

Evidence for associations of cholesterol and suicidality has also been reported in a series of studies in patients with various mental disorders revealing lower total cholesterol concentrations in suicide attempters (Golier, Marzuk, Leon et al., 1995; Kim, Lee, Kim et al., 2002; Kunugi, Takei, Aoki et al., 1997; Papassotiropoulos, Hawellek, Frahnert et al., 1999). However, these studies are based on small samples and lack control for confounding variables such as depression-related malnutrition and weight-loss, age, gender, dietary habits, alcohol consumption, physical activity, and medication effects (review: Brunner, Parhofer, Schwandt et al., 2002).

Epidemiological studies in the community, which are usually less vulnerable towards confounding factors typical for clinical studies, focused exclusively on completed suicide. The results of these studies are controversial. Some studies reported an association between lower cholesterol and completed suicide (Partonen, Haukka, Virtamo et al., 1999; Zureik, Courbon \& Ducimetière, 1996). Another study, however, failed to find such an association (Vartiainen, Puska, Pekkanen et al., 1994). Two studies reported a positive association between cholesterol and suicide (Iribarren, Reed, Wergowske et al., 1995; Tanskanen, Vartiainen, Tuomilehto et al., 2000). No data from general population samples are available 
on the relationship between suicide attempts and suicidal ideation and parameters of cholesterol and lipid metabolism.

A further shortcoming of epidemiological studies reporting associations of cholesterol with unfavorable health outcomes is that they focused almost exclusively on highly selective cohorts of subjects at risk for cardiovascular mortality and morbidity possibly including selection bias. Avoiding this potential limitation, the present study examines whether positive associations of major depressive episode (MDE), suicide attempts, and suicidal ideation can be established with parameters of cholesterol and lipid metabolism in an unselected nationally representative community sample of $\mathrm{n}=4,181$ subjects in the community (German National Health Interview and Examination Survey).

\section{METHOD}

\section{German National Health Interview and Examination Survey (GHS) and its Mental Health Supplement (GHS-MHS)}

The GHS is a nationwide epidemiological study of both somatic and mental health in Germany in a representative adult sample in the community (for detailed description see Jacobi, Wittchen, Müller et al., 2002). In brief, it consists of the social and somatic core survey (GHS-CS) and the Mental Health Supplement (GHS-MHS). Out of the core survey participants $(\mathrm{n}=7,124$, response rate $61.4 \%)$, a subsample was additionally interviewed for mental disorders. Respondents of the core survey older than 65 years were excluded because the psychometric properties of the CIDI, the interview used in the study, have not yet been satisfactorily established for use in older populations (Knäuper \& Wittchen, 1994). The conditional response rate of the GHS-MHS was $87.6 \%$, resulting in a total of 4,181 respondents who completed the mental health assessment. In order to adjust the net sample to German age, sex and community distribution, data were weighted for age, sex, and region corresponding to the national administrative statistics of December 1997. The presented weighted results can be regarded as representative for the German noninstutionalized population from 18 to 65 years of age (mean age: 42 years) with sufficient language skills to follow the interviews.

\section{Assessment of Somatic Diseases and Laboratory Parameters}

Diagnoses of somatic disorders were assigned on a composite approach including self-report measures, a standardized clinical interview by the medical doctor, and laboratory measures. Fasting blood samples were drawn on the day of the standardized medical interview (for detailed description see Thierfelder, Seher, \& Thefeld, 1998). The following parameters were considered for the subsequent analyses: total serum cholesterol, HDL cholesterol, triglycerides, BMI, age, and sex.

\section{Psychopathological Assessments}

Psychopathological assessments were based on a standardized diagnostic interview according to DSM-IV criteria (DIA-X/M-CIDI, Wittchen \& Pfister, 1997), administered by trained clinical interviewers. Diagnoses were computed using the CIDI/DSM-IV algorithm. Suicidality was assessed with the series of CIDI suicidality questions that were presented as part of the depression section. These questions comprise a series of six questions summarized in the footnote of Table 1. It should be noted that all the suicidality items were only asked to 
respondents who had acknowledged at least one of the core depression items. Thus, detailed results for the suicidality items were available for 1,205 individuals ( $26 \%$ of the total sample). In the text we present the prevalence of each of the suicidality items for the total sample $(\mathrm{n}=$ 4,181 ), those without major depressive episode (MDE, $n=3,789$ ) as well as subjects with $\operatorname{MDE}(\mathrm{n}=392,9.4 \%)$.

\section{Statistical Analysis}

Findings were adjusted for age and sex in all analyses to control for potential confounding by these factors. To account for the weighting scheme as well as the stratified sampling design by screening status (Jacobi, Wittchen, Müller et al., 2002), statistical inference (standard errors, confidence intervals, and p-values) was based on the Huber-White sandwich estimator of variance (Royall, 1986) using the Stata software package, release 7.0 (StataCorp, 2001). The associations of suicide attempts, suicidal ideation with BMI, triglycerides, and cholesterol were based on odds ratios estimated from logistic regression. For the association analyses, the covariates were standardized (mean $=0$, standard deviation $[\mathrm{SD}]=1$ ). Here, odds ratios indicate the increase of the odds for suicidality by increase of the covariates by one SD. Furthermore, comparisons of low (below the 25th percentile), normal (25th-75th percentile) and high (above the 75th percentile) values of the covariates were conducted.

\section{RESULTS}

\section{2-Month Prevalance of Suicidal Ideation and Suicide Attempts}

Table 1 shows the 12-month prevalence of suicidal ideation and suicide attempts in the total sample $(n=4,181)$ and among subjects with $(n=392,9.4 \%)$ and without $(n=3,789)$ MDE during the past 12 months. Of the general population, $11.7 \%$ reported having had a period where most of the time they were „thinking a lot about death.“ More specific suicidal ideation items were considerably less frequent with prevalences ranging from 3.8 (,feeling like wanting to die") to 1.2 (,planning to commit suicide"). The prevalence of attempted suicide during the past 12 months was 0.4 in the total sample.

Splitting the total sample $(\mathrm{n}=4,181)$ into those subjects with $(\mathrm{n}=392,9.4 \%)$ and without (n $=3,789$ ) a major depressive episode (MDE) during the past 12 months revealed that most of the prevalence in the total sample was due to subjects with MDE. Among the 392 subjects with MDE, $63 \%$ reported at least 2 weeks where they were ,thinking a lot about death,“ $31 \%$ reported a period of 2 weeks where they were ,feeling like wanting to die, “ $23 \%$ were thinking about committing suicide, and $11 \%$ were planning to commit suicide. The selfreported prevalence for attempted suicide was 3.4\% in subjects with MDE. The presence of MDE was associated with a considerably increased risk to report at least one suicidal item (odds ratios ranging from 21.8-33.6). It should be noted, however, that suicidality items were only assessed in those subjects who had reported at least one core depression item during the past 12 months $(\mathrm{n}=1,205)$.

In order to exclude the effect of comorbidity with psychoactive substance use disorder we analysed the association between a 12-month diagnosis of MDE and the 12-month diagnoses of nicotine dependence, alcohol abuse or dependence, and any illicit drug use disorder (abuse or dependence) among $\mathrm{n}=1,205$ cases with depressive symptoms in the past 12 months. No association between MDE and psychoactive substance use disorder in this subsample was found. Although suicidal ideation as well as suicide attempts tended to be more frequent 
among those with substance use diagnoses, the only significant associations (after adjusting for sex and age) were found between illicit drug abuse/dependence and ,planning to commit suicide" $(\mathrm{OR}=13.41,95 \% \mathrm{CI}=2.08-86.42)$ and between illicit drug abuse/dependence, and „thinking about committing suicide“ $(\mathrm{OR}=7.50,95 \% \mathrm{CI}=1.17-48.23)$.

\section{Association between Cholesterol, Triglycerides, BMI, and Suicidality}

Logistic regression analysis (Table 2) revealed positive associations of total cholesterol and triglycerides with some measures of suicidality (,thinking a lot about death“ and ,attempted suicide") in subjects with depressive symptoms during the past 12 months $(\mathrm{n}=1,205)$. It is noteworthy that despite the low rate for attempted suicide, the association reached statistical significance, whereas other items of suicidal ideation (,feeling like wanting to die, “",thinking about committing suicide," „planning to commit suicide“) did not. Furthermore, BMI was positively associated with attempted suicide.

To assess whether our findings could be explained by substance use disorders we repeated the analyses in Table 2 by also adjusting for 12 months nicotine dependence, alcohol abuse or dependence, and illicit drug abuse or dependence (the latter had to be omitted for the item „thinking a lot about death“ because of empty cells). The point estimates of odds ratios remained almost equal (data not shown) although the p-values for the associations between „triglycerides“ and „thinking a lot about death“( $\mathrm{OR}=1.30,0.996-1.69)$, and between ,total cholesterol“ and ,suicide attempts“ $(\mathrm{OR}=1.97,0.98-3.59)$ moved from barely under the 0.05 threshold to barely over the 0.05 threshold.

The covariates total cholesterol, triglycerides, and BMI were then split by the 25th and the 75th percentile and the three groups were compared after adjustment for age and gender effects (Table 3 ). Table 3 shows that subjects with depressive symptoms $(n=1,205)$ above the 75 th percentile had a more than fourfold increased odds for attempted suicide compared to the those below the 25 th percentile.

\section{DISCUSSION}

The aim of this study was to investigate the association between suicidality (suicidal ideation and suicide attempts), major depressive episode (MDE), and parameters of cholesterol and lipid metabolism in a community sample. This is, to our knowledge, the first epidemiological study in a representative community sample to examine whether cholesterol concentrations are related to increased suicidal behavior. The strengths of the study are: the sample size, detailed psychopathological data, and detailed self-report information about suicidal ideation and behavior.

The 12-month prevalence of MDE was $9.4 \%$ in this nationally representative sample of subjects aged $18-65$ years. The 12 -month prevalence of attempted suicide was $0.4 \%$ in the total sample $(n=4,181)$, but it is noteworthy that indicators of suicidal ideation were much more frequent. The proportion of all indicators of suicidality among those subjects with MDE was as expected considerably higher with rates of attempted suicide of $3.4 \%$. The presence of MDE was associated with a considerably increased risk for all suicidality items with odds ratios ranging from 21.8-33.6. These findings are well in accordance with previous studies, both for depression (Haarasilta, Marttunen, Kaprio et al., 2001; Lindeman, Hamalainen, Isometsä et al., 2000) as well as suicidal ideation (Weissman, Bland, Canino et al., 1999). The well known increased odds ratio of suicidal ideation with the presence of major depression is 
in line with previous studies (Dyck, Bland, Newman et al., 1988; Wunderlich, Bronisch, \& Wittchen, 1998). The findings of our study cannot be explained by comorbidity of psychoactive substance use disorder. No association between MDE in the last 12 months and psychoactive use disorder (12 months diagnoses of nicotine dependence, alcohol abuse or dependence, any illicit drug abuse or dependence) was found in our sample.

The key finding of this study is that total cholesterol and triglycerides showed a positive association with attempted suicide and, thinking a lot about death" in subjects with depressive symptoms during the past 12 months versus subjects with major depression without suicide attempts. In contrast to previous studies however, low cholesterol concentrations $(<5.5$ $\mathrm{mmol} / \mathrm{l}$ ) had a diminished OR for attempted suicide as compared to normal cholesterol concentrations, and the BMI was positively associated with attempted suicide. These findings are in contrast to some clinical studies in psychiatric patients (review: Brunner, Parhofer, Schwandt et al., 2002) as well as some previous epidemiological findings (Partonen, Haukka, Virtamo et al., 1999; Zureik, Courbon, \& Ducimetière, 1996). Although suicidal ideation and suicde attempts tended to be more frequent in our sample among those subjects with substance use diagnoses, the only significant associations (after adjusting for sex and age) were found between illicit drug abuse/dependence and ,planning to commit suicide“ and between illicit drug abuse/dependence and „thinking about committing suicide. “ It is difficult to explain this divergence of findings, however, design issues seem to primarily account for these differences: (1) Previous epidemiological findings refer to cohort studies of cardiovascular morbidity and mortality, focussing exclusively on completed suicide. Thus, these studies related cholesterol measurements to the rare event of subsequent completed suicide occurring years or even decades later and did not assess suicidal ideation or attempted suicide. (2) Further, results of the available five studies differ considerably. Two studies reporting lower cholesterol in suicide victims only included data for men (Partonen, Haukka, Virtamo et al., 1999; Zureik, Courbon, \& Ducimetière, 1996). In a cohort of 10,898 men and 11,534 women, no association between cholesterol and completed suicide was found (Vartiainen, Puska, Pekkanen et al., 1994). Iribarren, Reed, Wergowske et al. (1995) reported a positive association between cholesterol and risk of suicide in a sample of 7,309 men (24 suicides after 23 years of follow-up). The relative risk of suicide associated with an increment of $0.98 \mathrm{mmol} / 1$ in serum cholesterol was 1.46 in their study. In a population of 37,635 persons, Tanskanen and colleagues (2000) found that serum cholesterol was positively related to the risk of violent suicide. Among subjects whose total serum cholesterol was in the highest category $(>8.0 \mathrm{mmol} / \mathrm{l})$, the adjusted relative risk of violent suicide was more than twofold compared with the lowest category $(<5.0 \mathrm{mmol} / \mathrm{l})$.

The results of the present study reveal—when splitting the covariates total cholesterol, triglycerides, and BMI by the 25 th and the 75 th percentile and after adjustment for age and gender effects - that those subjects with depressive symptoms above the 75 th percentile had a more than fourfold increased odds for attempted suicide as compared to those below the 25th percentile. Subjects with high cholesterol concentrations $(>6.42 \mathrm{mmol} / \mathrm{l})$ showed an OR for attempted suicide of 4.5 as compared to low cholesterol concentrations $(<5.5 \mathrm{mmol} / \mathrm{l})$. These findings regarding attempted suicide are consistent with the two epidemiological studies reporting a positive association between cholesterol and completed suicide (Iribarren, Reed, Wergowske et al., 1995; Tanskanen, Vartiainen, Tuomilehto et al., 2000). However, some clinical studies in psychiatric patients with different diagnoses found lower cholesterol concentrations in suicide attempters (Golier, Marzuk, Leon et al., 1995; Kim, Lee, Kim et al., 2002; Kunugi, Takei, Aoki et al., 1997; Muldoon, Manuck, Matthews et al., 1990, Papassotiropoulos, Hawellek, Frahnert et al., 1999). The reasons behind this association are 
not clear. Several possible confounders have to be discussed (Brunner, Parhofer, Schwandt et al., 2002).

The majority of clinical studies in psychiatric patients and epidemiological studies only measured total cholesterol. The results of the present study show that the findings are not specific for cholesterol since both high triglycerides and BMI were also positively associated with attempted suicide. These findings are in line with the hypothesis that cholesterol may be of secondary physiological relevance and that for example polyunsatured fatty acids may be a critical confounding factor in the association between cholesterol and suicide (review:

Brunner, Parhofer, Schwandt et al., 2002). We speculate that a considerable amount of patients with higher BMI, triglycerides, and cholesterol may have maladaptive nutritional behaviors (such as binge eating). An association between binge eating and suicide attempts and suicidal ideation has been described in the literature (Favaro \& Santonastaso, 1997; Viesselman \& Roig, 1985). An alternative explanation could be a higher proportion of atypical depression with hyperphagia and weight gain. Another hypothesis would be that patients with overweight have more somatic comorbidity which leads to reduced quality of life. Some exploratory analysis in the present data set seemed to suggest some preliminary evidence for this assumption (data available on request). A limitation of the present study is that we cannot exclude possible confounding by antidepressant medication. It is described in the literature that mirtazapine increases total cholesterol concentrations and body weight in healthy subjects (Nicholas, Ford, Esposito et al., 2003). However, since only a few of the subjects in the community studies were on any antidepressant medication during the evaluation period, it is unlikely that this factor also accounts for the effect.

\section{CONCLUSION}

In conclusion, a positive, but moderate association between total cholesterol, triglycerides, BMI, and suicide attempts was found in subjects with depressive symptoms in the past 12 months. The results of this study are compatible with two recent epidemiological cohort studies showing a positive association between cholesterol and completed suicide (Iribarren, Reed, Wergowske et al., 1995; Tanskanen, Vartiainen, Tuomilehto et al., 2000). Our study does not reveal any support for the for the hypothesis that cholesterol-lowering therapies or naturally occurring low cholesterol concentrations are associated with increased risk of attempted suicide. However, further studies should continue to monitor this issue.

TABLE 1. 12-Month Prevalence of Suicidal Ideation and Suicide Attempts

\begin{tabular}{|c|c|c|c|c|c|c|c|c|c|c|c|}
\hline & \multicolumn{3}{|c|}{$\begin{array}{l}\text { Total sample } \\
(\mathrm{n}=4181)\end{array}$} & \multicolumn{3}{|c|}{$\begin{array}{l}\text { Without } \mathrm{MDE}^{a} \\
(\mathrm{n}=3789)\end{array}$} & \multicolumn{5}{|c|}{$\begin{array}{l}\text { Major depressive episode } \\
\quad(\mathrm{MDE})^{a}(\mathrm{n}=392)\end{array}$} \\
\hline & $\mathbf{n}$ & $\%$ & $95 \% \mathrm{CI}$ & $\mathbf{n}$ & $\%$ & $95 \% \mathrm{CI}$ & $\mathbf{n}$ & $\%$ & $95 \% \mathrm{CI}$ & $\mathrm{OR}^{b}$ & $95 \% \mathrm{CI}$ \\
\hline \multicolumn{12}{|l|}{ Suicidal ideation } \\
\hline $\begin{array}{l}\text { Thinking a bot about } \\
\text { death }\end{array}$ & 490 & 11.7 & $10.7-12.8$ & 302 & 7.0 & $6.2-7.9$ & 248 & 63.3 & $57.8-68.4$ & $21.8^{* * *}$ & $16.7-28.5$ \\
\hline Feeling like wanting to die & 157 & 3.8 & $3.2-4.4$ & 60 & 1.3 & $1.0-1.7$ & 119 & 30.9 & $25.9-36.3$ & $33.4^{* * *}$ & $22.8-48.8$ \\
\hline $\begin{array}{l}\text { Thinking about } \\
\text { committing suicide }\end{array}$ & 121 & 29 & $2.4-3.5$ & 50 & 1.0 & $0.7-1.4$ & 88 & 23.4 & $18.9-28.5$ & $29.2^{* *}$ & $19.1-44.6$ \\
\hline Panning to commit suicide & 50 & 1.2 & $0.9-1.6$ & 18 & 0.3 & $0.2-0.60$ & 45 & 10.6 & $7.7-14.5$ & $33.6^{* * *}$ & $17.5-64.5$ \\
\hline Attempted suicide & 16 & 0.4 & $0.2-0.6$ & 7 & 0.1 & $0.0-0.2$ & 14 & 3.4 & $20-5.9$ & $32.2^{* * *}$ & $10.8-95.5$ \\
\hline
\end{tabular}




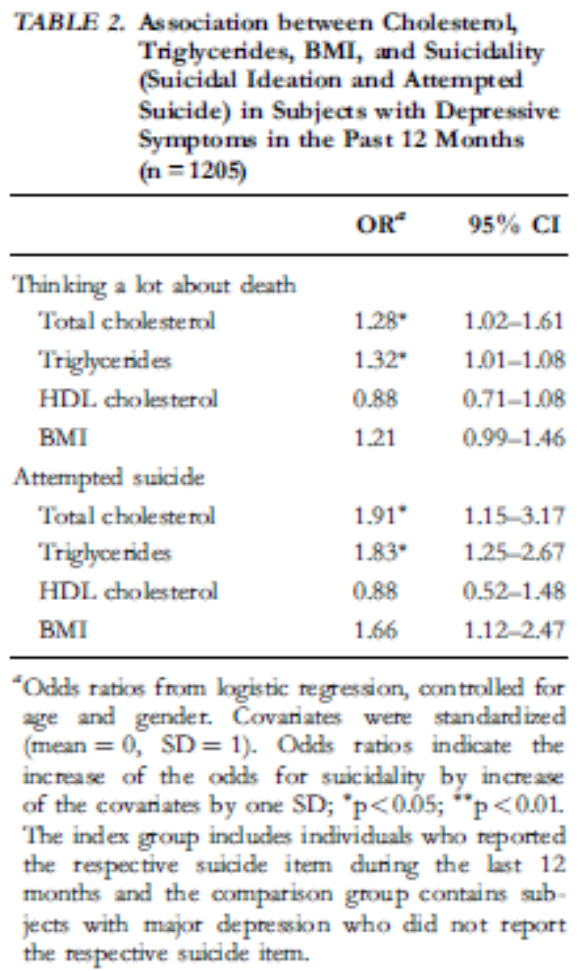

TABLE 3. Association between Attempted Suicide and Total Cholesterol, Triglycerides, HDL Cholesterol, and BMI in Subjects with Depressive Symptoms in the Past 12 Months $(n=1205)$

\begin{tabular}{|c|c|c|c|c|c|c|}
\hline & \multicolumn{6}{|c|}{ Association of suicide attempts by reference groups } \\
\hline & \multicolumn{2}{|c|}{ bw vs. normal } & \multicolumn{2}{|c|}{ high vs. normal } & \multicolumn{2}{|c|}{ high vs. low } \\
\hline & $\mathrm{OR}^{2}$ & $95 \% \mathrm{CI}$ & $\mathrm{OR}^{a}$ & $95 \%$ CI & $\mathrm{OR}^{2}$ & $95 \% \mathrm{CI}$ \\
\hline Total cholesterol & $0.30^{\circ}$ & $0.09-0.99$ & 1.37 & $0.38-4.93^{*}$ & $4.50^{\circ}$ & $1.09-18.61$ \\
\hline Trigycerides & 0.76 & $0.25-2.35$ & 3.23 & $0.89-11.67$ & $4.25^{*}$ & $1.30-13.91$ \\
\hline HDL cholesterol & 1.79 & $0.54-5.98$ & 1.04 & $0.29-3.65$ & 0.58 & $0.15-2.31$ \\
\hline BMI & 0.68 & $0.19-2.43$ & $3.75^{\circ}$ & $1.06-13.27$ & $5.50^{\circ}$ & $1.38-21.96$ \\
\hline
\end{tabular}

"Odds ratios from logistic regression, controlled for age and gender. ORs representcomparisons of low (below the 25 th percentile), normal (25th-75thpercentile) and high (above the 75 th percentile) val ues of the covariates. Cholesterol: low $<5.5 \mathrm{mmol} / \mathrm{l}$, normal $5.5-6.42 \mathrm{mmol} / \mathrm{l}$, high $>6.42 \mathrm{mmol} / \mathrm{L}$. Triglycerides: low $<0.81 \mathrm{mmol} / 1$, normal 0.81-2.25 mmol/1, high $>2.25 \mathrm{mmol} / \mathrm{L}$. BMI: low $<23.7$, normal $23.7-31.8$, high $>31.8$. The index group includes individuals who reported sucide attempts during the past 12 months and the comparison group contains subjects with major depression who did not report suicide attempts.

P $<0.05$.

\section{AUTHOR NOTE}

Jürgen Brunner, Thomas Bronisch, Hildegard Pfister, and Michael Höfler, Max Planck Institute of Psychiatry, Munich, Germany. Frank Jacobi, Institute of Clinical Psychology and Psychotherapy, Technical University of Dresden, Germany. Hans-Ulrich Wittchen, Max Planck Institute of Psychiatry, Munich, Germany and Institute of Clinical Psychology and Psychotherapy, Technical University of Dresden, Germany.

This study was supported by grant 01EH970/8 (German Federal Ministry of Research, Education and Science, BMBF). Reported data on mental disorders were assessed in the 
Mental Health Supplement of the German Health Survey (GHS-MHS), conducted by the Max Planck Institute of Psychiatry, Munich. Principal investigator was Prof. Dr. Hans- Ulrich Wittchen. Laboratory and BMI data came from the core survey (GHS-CS), conducted by the Robert Koch Institute, Berlin. Principal investigators of the GHS-CS were Dr. Bärbel-Maria Kurth and Dr. Wolfgang Thefeld.

Correspondence concerning this article should be addressed to Jürgen Brunner, Max Planck Institute of Psychiatry, Kraepelinstr. 10, 80804 Munich, Germany. E-mail: jbrunner@mpipsykl.mpg.de.

\section{REFERENCES}

- Brunner, J., Parhofer, K. G., Schwandt, P., et al. (2002). Cholesterol, essential fatty acids, and suicide. Pharmacopsychiatry, 35, 1-5.

- $\quad$ Dyck, R. J., Bland, R. C., Newman, S. C., et al. (1988). Suicide attempts and psychiatric disorders in Edmonton. Acta Psychiatrica Scandinavica, 338,64-71.

- Favaro, A. \& Santonastaso, P. (1997). Suicidality in eating disorders: Clinical and psychological correlates. Acta Psychiatrica Scandinavica, 95, 508-514.

- Golier, J. A., Marzuk, P. M., Leon, A. C., et al. (1995). Low serum cholesterol level and attempted suicide. American Journal of Psychiatry, 152, 419-423.

- Haarasilta, L., Marttunen, M., Kaprio, J., et al. (2001). The 12-month prevalence and characteristics of major depressive episode in a representative nationwide sample of adolescents and young adults. Psychological Medicine, 31, 1169-1179.

- Iribarren, C., Reed, D. M., Wergowske, G., et al. (1995). Serum cholesterol level and mortality due to suicide and trauma in the Honolulu heart program. Archives of Internal Medicine, 155, 695-700.

- Jacobi, F., Wittchen, H.-U., Müller, N., et al. (2002). Estimating the prevalence of mental and somatic disorders in the community: Aims and methods of the German National Health Interview and Examination Survey. International Journal of Methods in Psychiatric Research, $11,1-19$.

- Kim, Y. K., Lee, H. J., Kim, J. Y., et al. (2002). Low serum cholesterol is correlated to suicidality in a Korean sample. Acta Psychiatrica Scandinavica, 105,141-148.

- Knäuper B. \& Wittchen H.-U. (1994). Diagnosing major depression in the elderly: Evidence for response bias in standardized diagnostic interviews? Journal of Psychiatric Research, 28, $147-164$.

- Kunugi, H., Takei, N., Aoki, H., et al. (1997). Low serum cholesterol in suicide attempters. Biological Psychiatry, 41, 196-200.

- LaRosa, J. C., He, J., \& Vupputuri, S. (1999). Effect of statins on risk of coronary disease. A metaanalysis of randomized controlled trials. Journal of the American Medical Association, $282,2340-2346$.

- Lindeman, S., Hamalainen, J., Isometsä, E., et al. (2000). The 12-month prevalence and risk factors for major depressive episode in Finland: Representative sample of 5993 adults. Acta Psychiatrica Scandinavica, 102, 178-184.

- Muldoon, M., Manuck, S. B., \& Matthews, K. A. (1990). Lowering cholesterol concentrations and mortality: A quantitative review of primary prevention trials. British Medical Journal, 301,309-314.

- Muldoon, M. F., Manuck, S. B., Mendelsohn, A. B., et al. (2001). Cholesterol reduction and non-illness mortality: Meta-analysis of randomised clinical trials. British Medical Journal, $322,11-15$.

- Nicholas, L. M., Ford, A. L., Esposito, S. M., et al. (2003). The effects of mirtazapine on plasma lipid profiles in healthy subjects. Journal of Clinical Psychiatry, 64: 883-889. 
- Papassotiropoulos, A., Hawellek, B., Frahnert, C., et al. (1999). The risk of acute suicidality in psychiatric inpatients increases with low plasma cholesterol. Pharmacopsychiatry, 32, 1-4.

- Partonen, T., Haukka, J., Virtamo, J., et al. (1999). Association of low serum total cholesterol with major depression and suicide. British Journal of Psychiatry, 175, 259-262.

- Royall, R. M. (1986). Model robust confidence intervals using maximum likelihood estimators. International Statistical Review, 54, 221-226.

- StataCorp. (2001). Stata Statistical Software: Release 7.0. College Station; TX: Stata Corporation.

- Tanskanen, A., Vartiainen, E., Tuomilehto, J., et al. (2000). High serum cholesterol and risk of suicide. American Journal of Psychiatry, 157, 648-650.

- Thierfelder, W., Seher, C., \& Thefeld, W. (1998) Der Bundes-Gesundheitssurvey 1997/98 Untersuchungsteil. Gesundheitswesen, 60, S69-S76.

- Vartiainen, E., Puska, P., Pekkanen, J., et al. (1994). Serum cholesterol concentrations and mortality from accidents, suicide, and other violent causes. British Medical Journal, 13, 445447.

- Viesselman, J. O. \& Roig, M. (1985). Depression and suicidality in eating disorders. Journal of Clinical Psychiatry, 46, 118-124.

- Weissman, M. M., Bland, R. C., Canino, G. J., et al. (1999). Prevalence of suicide ideation and suicide attempts in nine countries. Psychological Medicine, 29, 9-17.

- Wittchen H.-U. \& Pfister, H. (1997). DIA-X-Interviews: Manual für Screening-Verfahren und Interview; Interviewheft Längsschnittuntersuchung (DIA-X-Lifetime); Ergänzungsheft (DIAX-Lifetime); Interviewheft Querschnittuntersuchung (DIA-X-12 Monate); Ergänzungsheft (DIA-X-12 Monate); PC-Programm zur Durchführung des Interviews (Längs- und Querschnittuntersuchung); Auswertungsprogramm. Frankfurt: Swets \& Zeitlinger.

- Wunderlich, U., Bronisch, T., \& Wittchen, H.-U. (1998) Comorbidity patterns in adolescents and young adults with suicide attempts. European Archives of Psychiatry and Clinical Neuroscience, 248, 87-95.

- Zureik, M., Courbon, D., \& Ducimetière, P. (1996). Serum cholesterol concentration and death from suicide in men: Paris prospective study I. British Medical Journal, 313, 649-651. 